\title{
Review of: "VEGFR1 signaling in retinal angiogenesis and microinflammation"
}

\author{
Matthew Nugent ${ }^{1}$ \\ 1 University of Massachusetts at Lowell
}

Potential competing interests: The author(s) declared that no potential competing interests exist.

Uemura et al., have done a service to the field by providing a detailed and comprehensive review of the role of VEGF receptor 1 (VEGFR1) in retinal angiogenesis and microinflammation. The role of VEGFR1 is often simplified to that of a decoy receptor that sequesters VEGF-A and prevents it from activating its signaling receptor, VEGFR2. While this is certainly an important aspect of the function of VEGFR1 within the VEGF system, it is only a very small part of a much larger story. Complexity! Indeed, the sophistication and complexity of the VEGF system can be admittedly maddening and frustrating, leaving one to simply throw one's hands up in surrender. But, the authors of this review did not surrender, but instead they rolled up their sleeves and dug into the details. In so doing they have provided a wonderful survey of what is known and, importantly, what is left to be discovered. Toward this end, the authors are also careful to qualify their conclusions regarding how they believe the system works.

The role of VEGFR1 as a direct signaling receptor was, at one point, cast off as insignificant based on the seminal study by Hiratsuka et al., that showed that mice engineered to express a truncated form of VEGFR1 that lacked the tyrosine kinase domain are healthy and fertile. Yet, Uemura et al., provide interesting information that counters this simple conclusion. Indeed, they illustrate evidence that argues for important VEGFR1-mediated signaling events in a variety of normal and pathologic processes and are careful to describe potential explanations for what appear to be, at first blush, contradictory findings. This is a careful scientific approach. The challenge is that it makes for a very dense read, but one that is worth the effort. While the authors do an exceptional job of summarizing a rather large literature, one could quibble with the relatively limited description of the role of non-receptor interactions with VEGF ligands, particularly within the extracellular matrix. It might have been nice to extend some of these ideas to include how cells, VEGF, and VEGF receptors are interacting with proteins and proteoglycans in the matrix. Nevertheless, the authors provide an informative list of open questions that are ready for investigation, such as: careful and quantitative comparisons of various anti-VEF therapies, the relative interactions between VEGF-A, VEGF-B and PIGF, and the roles for ligand and receptor heterodimers. Indeed, I would recommend this review for a new PhD student or postdoc in search of an important scientific question to tackle in the VEGF field. The VEGF system is extremely complex, which should not come as a surprise considering its critical role in development, homeostasis and disease. The authors of this review, to their 
credit and to that of the field, did not shy away from this fact. As Einstein said, "Everything should be made as simple as possible, but not simpler." 